\title{
Insufficient evidence to support or refute the need for 6-monthly dental check-ups
}

\author{
What is the optimal recall frequency between dental checks?
}

\author{
Beirne P, Forgie A, Clarkson JE, Worthington HV. Recall \\ intervals for oral health in primary care patients. Cochrane \\ Database of Systematic Reviews 2005, Issue 2. Art. No.: \\ CD004346
}

Data sources Trials were sourced using the Cochrane Oral Health Group Trials Register, the Cochrane Central Register of Controlled Trials, Medline and Embase. Reference lists from relevant articles were scanned and the authors of some papers were contacted to identify further trials and obtain additional information.

Study selection Trials were selected if they met the following criteria:

design: random allocation of participants;

participants: all children and adults receiving dental check-ups in primary-care settings, irrespective of their level of risk for oral disease; interventions: recall intervals for either clinical examination only, clinical examination plus scale and polish, clinical examination plus preventive advice, clinical examination plus scale and polish plus preventive advice, no recall interval/patient-driven attendance (which may be symptomatic), or clinician risk-based recall intervals;

outcomes: clinical status outcomes for dental caries including, but not limited to, mean dmft/DMFT, dmfs/DMFS scores, caries increment, filled teeth (including replacement restorations), early carious lesions (arrested or reversed); periodontal disease (including, but not limited to, plaque, calculus, gingivitis, periodontitis, change in probing depth, and attachment level); oral mucosa (presence or absence of mucosal lesions, potentially malignant lesions, cancerous lesions, and size and stage of cancerous lesions at diagnosis).

In addition, the following outcomes were considered where reported: patient-centred outcomes, economic-cost outcomes, other outcomes such as improvements in oral health knowledge and attitudes, harms, changes in dietary habits, and any other oral health-related behavioural change.

Data extraction and synthesis Information regarding methods, participants, interventions, outcome measures, and results were independently extracted, in duplicate, by two authors. Authors were contacted, where deemed necessary and where possible, for further details regarding study design and for data clarification. A quality assessment of the included trial was carried out. The Cochrane Oral Health Group's statistical guidelines were followed.

Results Only one study (with 188 participants) was included in this review and was assessed as having a high risk of bias. This study provided limited data for dental caries outcomes (dmfs/DMFS increment) and economic cost outcomes (reported time taken to provide examinations and treatment).

Conclusions There is insufficient evidence from randomised controlled trials (RCT) to draw any conclusions regarding the potential beneficial and harmful effects of altering the recall interval between dental check-ups. There is insufficient evidence to support or refute the practice of encouraging patients to attend for dental check-ups at

Address for correspondence: Emma Tavender, Review Group Co-ordinator, Cochrane Oral Health Group, University Dental Hospital of Manchester, Higher Cambridge Street, Manchester M15 6FH, UK. E-mail: emma.tavender@man.ac.uk 6-monthly intervals. It is important that high quality RCT are conducted for the outcomes listed in this review in order to address its objectives.

\section{Commentary}

This systematic review addresses a relevant issue in oral health care. It has been consistently performed according to the high standards of the Cochrane Collaboration. The results are not really surprising and confirm the conclusions of a similar review. ${ }^{1}$

Both reviews underpin the paucity of clinical research evidence in this area (one RCT of poor quality). The (cost)-effectiveness of population-based screening procedures (eg, screening of breast cancer) is a subject of ongoing debate, so scientific evidence is therefore urgently required: general dental practitioners routinely perform check-ups in relatively healthy individuals regularly attending dental practice (for instance, in The Netherlands this is $80 \%$ of the population).

The reviewers highlight that there is no universally accepted definition of what constitutes a routine oral examination. They list five possible types of dental check-ups in their inclusion criteria: clinical examination only; clinical examination plus scale and polish; clinical examination plus preventive advice; clinical examination plus both and clinical examination based on the clinician's assessment of patient's risk.

Considering the increasingly prevention-orientated goal of regular attendance in dentistry, there is obviously no contemporary rationale for conducting ROE without incorporating preventive advice. Prevention encompasses not only screening but also oral health education advice and feedback. Oral health education advice (simple instruction on oral hygiene and recurrent promotion of fluoride-containing agents) has been shown to be beneficial, in the short term, to individual patients if it is provided in clinical practice. $^{2-4}$

According to "the implications for research" section of the review, more emphasis should be placed on defining and investigating what the most effective content of ROE should be. The current opinion is that it is preferable to prevent the onset of oral diseases by using individual risk profiles and tailored recall intervals. The recently published UK National Institute for Clinical Excellence guidance likewise promotes reviews of oral health status based on individual risk factors. ${ }^{5}$ One of the authors' recommendations here could have been that preventive advice should always be an essential part of ROE.

Surprisingly, opinions and needs of patients regarding the ROE and tailored recall intervals remain unknown and research on this topic should be encouraged. Research on complex multifaceted interventions such as the ROE is difficult, however, with the need for screening, diagnosis, and perhaps preventive interventions, for various conditions within different subgroups. RCT represent final tests by definition, whereas insight into the various components of the preventive performance, for example, content of oral examination, length of follow-up period, is too limited to define an optimal trial design. To distinguish small but relevant clinical and nonclinical effects for different recall periods, trials have to be conducted for relatively long periods and with sufficient partici- 
pants. Given the progression rates of multifactorial diseases such as dental caries and periodontal disease, loss of follow-up is an important threat to the validity of studies. The use of a generic clinical outcome measure, such as quality of adjusted life-years related to oral health, could help to overcome the problem of heterogeneous outcomes.

The Cochrane review must be credited for the comprehensive and meticulous research work of the authors, and their plea for a restart in population-based oral screening research in dentistry, despite the emerging research of the past decades. This creates a new challenge in designing and conducting reliable practice- and evidence-based research.

\section{Practice points}

- Fixed recall intervals (mostly 6 months) for all patients are not evidence-based.

- Before further evidence emerges, experienced practitioners could select low-risk individuals for extension of the recall period beyond the standard 6 months.

- Efforts should be made to improve clinical decision making focussed on people with elevated risk of oral disease, resulting in advanced preventive counselling and assessment of risk indicators, combined with systematic record keeping of clinical and nonclinical data.

\section{Dirk Mettes}

College of Oral Sciences, Radboud University Nijmegen (formerly University of Nijmegen) Medical Centre, Nijmegen, The Netherlands

1. Davenport C, Elly K, Salas C, Taylor-Weetman CL, Fry-Smith A, Bryan S. The clinical effectiveness and cost-effectiveness of routine dental checks: a systematic review and economic evaluation. Health Technol Assess 2003; 7.

2. Sprod AJ, Anderson R, Treasure ET. Effective Oral Health Promotion. Literature Review, Cardiff: Health Promotion Wales; 1996, Technical Report 20.

3. Kay E, Locker D. A systematic review of the effectiveness of health promotion aimed at improving oral health. Commun Dent Health 1998; 15:132-144.

4. Kressin NR, Boehmer U, Nunn ME, Spiro III A. Increased preventive practices lead to greater tooth retention. I Dent Res 2003; 82:223-227.

5. Dental Recall. Recall Intervals between Routine Dental Examinations, London: National Institute of Clinical Excellence; 2004, Clinical guideline no. 19.

Evidence-Based Dentistry (2005) 6, 62-63.

doi:10.1038/sj.ebd.6400341 